\title{
Learning from Adversity: Lessons from the COVID-19 Crisis
}
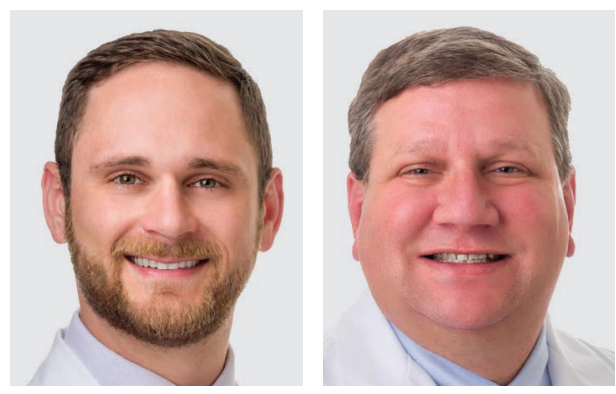

One month into the crisis caused by COVID-19 (coronavirus disease 2019; the disease caused by SARS-CoV-2) in the United States, rheumatologists began to feel the effects of our hastily constructed temporary response plans. While we may not have been staffing the front lines with our colleagues in emergency medicine and critical care, our patients were uniquely vulnerable to infection and continued to require care. Managing their illnesses remained no less important then than it ever was. Necessity forced us to learn important lessons about delivering this care in safer, more efficient ways. While some of these adaptations will revert to "business as usual," others may change the way we communicate with patients and colleagues long after this crisis has passed.

In our own clinic, the importance of social distancing catalyzed a transition to telemedicine. Healthcare has typically been excluded from stay-at-home orders, so patients can still come into the clinic, but should they? Many of us practice in large medical complexes. Reducing traffic in these locations makes social and medical sense. Even for rheumatologists with independent offices, waiting rooms are a worrisome potential locus for virus transmission. Most importantly, physicians and medical staff have close personal contact with dozens of patients per day, many of whom could be asymptomatic carriers. The rationale for moving to telemedicine was strong.

Telemedicine is not new to rheumatology. Publications on telemedicine approaches go back 20 years, though the major focus has typically been on the use of these tools to deliver specialty care to rural areas ${ }^{1,2}$. Despite this, adoption has been slow, and in many places, nonexistent. Many factors have contributed. First, technical barriers exist, and clinicians have worried about having the right tools. Second, financial factors have held back widespread adoption. Third, concerns about liability have made even the most tech-savvy suspicious. Finally, many fear that adopting distance rheumatology approaches will erode the doctor-patient relationship. Our experience has given us hope that many of these concerns are overstated.

Technical barriers were the easiest to overcome. Many of the structures required to convert to telemedicine have already been built into existing systems. Our academic medical practice had been considering telemedicine for years, and our electronic medical record system already contained ready-made workflows. When this crisis hit, we moved to an entirely virtual rheumatology clinical practice within a week. Clinician concerns about sharing their personal contact information were alleviated by other novel tools, such as the Doximity app, which allows them to call from their mobile phones without making the number recognizable to patients. This app even allows for conference calling, and facilitating faculty supervision of trainee phone visits in real time.

Financial factors have been an impediment to telemedicine as well. In the United States, prior guidance from the Centers for Medicare \& Medicaid Services (CMS) has restricted coverage for telemedicine to patients in rural areas. With the declaration of the state of emergency related to the COVID-19 pandemic, CMS announced a waiver that allows telemedicine to be covered more generally ${ }^{3}$. Many private insurers are following suit. This waiver covers a broad array of technologies for delivering telemedicine services and has reassured clinicians that they will be reimbursed for these services. If this were to survive the COVID-19 crisis in some form, financial impediments to telemedicine may be reduced or removed entirely.

Liability issues have been an important consideration for telemedicine services in the past, given the unclear legal implications of delivering services across state lines. In the current situation, where telemedicine has been rolled out within our practice area, this has been less of a concern. The CMS waiver has also made it clear that, at least for now, these encounters are being treated as an equivalent alternative to an in-person visit. Our hospital has even embraced the concept of telemedicine consultations for inpatients, a heretofore unthinkable practice. Thus far, these have been delivered to the satisfaction of both the requesting and the consulting service.

Most importantly, the response to the outpatient telemedicine visits among our patients has been overwhelmingly positive. Patients appreciate the chance to avoid traveling and have been grateful to speak with their provider without being exposed to potential risk from COVID-19. Clinicians were initially apprehensive, but many have realized that a large number of visits can be done safely without physical contact. Of course, part of this may be due to their expectation that in-person encounters will resume shortly. And for some

Personal non-commercial use only. The Journal of Rheumatology Copyright $(\subset) 2020$. All rights reserved. 
patients, particularly new patients, the inability to do a physical examination creates a great deal of uncertainty around the medical advice that can be delivered. Video approaches to telemedicine may alleviate this concern to some degree, but seem unlikely to eliminate it entirely. Clinicians are used to what they know, and what they have always known is that the physical examination is a key element of their assessment. It is called a history and a "physical" for a reason.

Despite these successes, we have encountered a number of unforeseen obstacles. Infusion therapies have been particularly challenging, as these cannot be delivered remotely and frequently involve our most immunosuppressed patients. In our clinic, we first initiated strict pre-appointment risk screening. Patients with fever or symptoms were asked to reschedule, both to avoid continuing immunomodulatory therapy in the setting of potential COVID-19 infection and also to avoid risk to other patients. We also implemented a personal protective equipment policy, using procedure masks for both patients and providers. To further reduce the risk of spread, we removed 2 of our 8 chairs, ensuring that we maintain a 6-foot distance between patients. To make up for the reduced capacity, we have shortened infusion times when able and stretched the scheduled infusion intervals for stable patients. Close monitoring will be necessary to ensure that these patients remain in good disease control.

We should note that the effect of the pandemic on academic medicine has extended far beyond clinical care. While data analysis, grant writing, and manuscript preparation can be done remotely, that is not the case for laboratory research, cohort studies, and randomized clinical trials. Many clinicians report feeling isolated, missing the camaraderie of caring for patients within their group practices. Web conferences have been helpful for many; ours holds twice-weekly Zoom meetings to connect. These will never replace in-person contact, and along with many others we will welcome the return of our bustling workroom.

Just as social distancing facilitated a transition to telemedicine, Web conferences have opened up new avenues for interinstitutional collaboration. If all of your meetings must be on Zoom, they may as well include physicians from New Zealand. Two examples highlight the diversity of our recent experience. First, after realizing that rheumatology patients were not specifically identified in many cohort studies, an international group of rheumatologists formed the COVID-19 Global Rheumatology Alliance (rheum-covid. org). Within 2 weeks of inception, the study has enrolled over 50 rheumatology patients with COVID-19 infections from academic medical centers around the world. Second, the struggle faced by rheumatology program directors with the challenges of didactic learning in the face of social distancing has brought about the Virtual Rheumatology Learning Collaborative, a lecture series for rheumatology programs across the United States. Rheumatology fellows will continue to learn remotely and will have the additional benefit of exposure to experts from outside their own institution.

Many of the changes driven by this crisis will pass, but some degree of telemedicine is likely here to stay. Patients, clinicians, and insurers will have recognized the convenience of this approach, and we suspect that some elements will be integrated into our practices going forward. To properly assess the effect of telemedicine, good data will be necessary. Controlled trials of telemedicine for specific circumstances will be warranted. Web conferencing may also outlive the pandemic, as so many of us realize the benefits of collaborating with colleagues across the world from the comfort of our living rooms. In the moment, it is hard to see anything positive in the tragedy of this pandemic. We will eventually come through it, though, and may take some solace in the hope that constructive changes may follow us to the other side.

\section{MICHAEL S. PUTMAN ${ }^{D}$, MD,}

Northwestern University Feinberg School of Medicine, Division of Rheumatology;

ERIC M. RUDERMAN, MD,

Northwestern University Feinberg School of Medicine, Division of Rheumatology,

Chicago, Illinois, USA.

Address correspondence to Dr. E.M. Ruderman, Northwestern University Feinberg School of Medicine, Division of Rheumatology, 675 North St. Clair 14-100, Chicago, Illinois 60611, USA.

E-mail: e-ruderman@northwestern.edu

\section{REFERENCES}

1. McDougall JA, Ferucci ED, Glover J, Fraenkel L. Telerheumatology: a systematic review. Arthritis Care Res 2017;69:1546-57.

2. Piga M, Cangemi I, Mathieu A, Cauli A. Telemedicine for patients with rheumatic diseases: systematic review and proposal for research agenda. Semin Arthritis Rheum 2017;47:121-8.

3. Centers for Medicare \& Medicaid Services. Medicare telemedicine health care provider fact sheet. [Internet. Accessed April 8, 2020.] Available from: www.cms.gov/newsroom/fact-sheets/ medicare-telemedicine-health-care-provider-fact-sheet

First Release May 1 2020; J Rheumatol 2020;47:791-2; doi:10.3899/jrheum.200411 